\title{
Prediction of the anomalous magnetic moment of nucleon from the nucleon anomaly
}

\author{
Yeu-Chung Lin \\ Department of Physics, National Central University, Chung-Li, Taiwan 32054
}

(November 18, 2018)

\begin{abstract}
We construct the effective anomaly lagrangian involving nucleons and photons by using current-current coupling method. The contribution of this lagrangian to the anomalous magnetic moment of nucleon is purely isovector. The anomalous magnetic moment of proton, $\kappa_{P}$, can be calculated from the this lagrangian and it is found to be $\kappa_{P}^{\text {Theor. }}=1.77$, which is in excellent agreement with the experimental value $\kappa_{P}^{E x p}=1.79$. While the case of neutron, $\kappa_{N}^{T h e o r .}=-2.58$ as compared to $\kappa_{N}^{E x p}=-1.91$, is less satisfactory, but the sign is correct.
\end{abstract}


Introduction The Skyrme model [1] reveals the underlying $Q C D$ physics of baryon in the large $N_{c}$ limit by considering baryon as a soliton made of mesons. The Skyrme lagrangian, inheriting it's form from the meson lagrangian, contains two parts, the normal parity part, which is basically the kinetic energy term, and the abnormal parity part, which is known as W.Z.W. [2.3] terms. The electromagnetic interaction can be incorporated into the Skyrme lagrangian by the same way as what the meson lagrangian does. It is interesting to observe that both the $\gamma P P$ and $\gamma \gamma P P$ vertices appear in both normal and abnormal parity parts of Skyrme lagrangian, as contrary to what we learned from the meson lagrangian that amplitudes never receive mixed contribution from different parity sectors. The reason for this difference is quite simple. The notion of $G$-parity failes to work for baryon, and hence the distinction between normal and abnormal parity is meaningless in the baryon case. Nevertheless, the existence of $\gamma P P$ and $\gamma \gamma P P$ vertices in the W.Z.W. terms indicates that these two vertices could receive contribution from anomaly. It is the main goal of the present work to construct an effective lagrangian to describe the anomaly effect which contributes to $\gamma P P$ and $\gamma \gamma P P$ vertices. And then use the lagrangian so constructed to predict the anomalous magnetic moment of proton.

Construction of Effective Lagrangian A heuristic way for the construction is to consider the formation of the triangle anomaly. The structure of triangle anomaly is determined by the triangle loop and the parity of the $A V V$ external currents. It is insensitive to the actual contents of the external currents. Replacement of one of the photon by $Z$ (which also contains a vector part) in $\pi^{0} \rightarrow \gamma \gamma$, for example, will not spoil the fact of being an anomaly.

Inspired by the above observation, we can use the $\pi^{0} \rightarrow \gamma \gamma$ anomaly as the building block of the construction of effective lagrangian involving nucleons. Namely, we can cast the amplitude of $\pi^{0} \rightarrow \gamma \gamma$ anomaly into a current-current coupling form

$$
A\left(\pi^{0} \rightarrow \gamma \gamma\right)=-f_{\pi} \partial_{\mu} \pi^{0} \cdot \frac{e^{2}}{4 \pi^{2} f_{\pi}^{2}} \epsilon^{\alpha \beta \mu \nu} A_{\nu} \partial_{\alpha} A_{\beta},
$$

and then replace the pion axial current by the nucleon axial current. The result of this 
substitution can be used to describe the nucleon anomaly. The relative normalization of the neutral axial currents of pion and nucleon is determined by the axial current derived from the $\pi$-N lagrangian. The neutral axial current is given by

$$
J_{\mu}^{5}=-f_{\pi} \partial_{\mu} \pi^{0}+\bar{\psi} \gamma_{\mu} \gamma_{5} \frac{\tau^{3}}{2} \psi
$$

Replacing the pion current in eq. 1 by the nucleon current in eq. 2, we derive a piece in the effective lagrangian which describes the $\gamma \gamma P P$ interaction

$$
L_{P P \gamma \gamma}=\frac{e^{2}}{4 \pi^{2} f_{\pi}^{2}} \epsilon^{\alpha \beta \mu \nu} \bar{\psi} \gamma_{\mu} \gamma_{5} \frac{\tau^{3}}{2} \psi A_{\nu} \partial_{\alpha} A_{\beta} .
$$

This piece of lagrangian is obvious not gauge invariant by itself. By trial and error, we can add another piece to make it gauge invariant

$$
\begin{aligned}
L= & \frac{e^{2}}{4 \pi^{2} f_{\pi}^{2}} \epsilon_{\alpha \beta \mu \nu} \bar{\psi} \gamma_{\mu} \gamma_{5} \frac{\tau^{3}}{2} \psi A_{\nu} \partial_{\alpha} A_{\beta} \\
& +\frac{e}{i 4 \pi^{2} f_{\pi}^{2}} \epsilon_{\alpha \beta \mu \nu} \bar{\psi} \gamma_{\mu} \gamma_{5} \frac{\tau^{3}}{2} \partial_{\nu} \psi \partial_{\alpha} A_{\beta} .
\end{aligned}
$$

The effective lagrangian so constructed describes the anomalous interactions of nucleons and photons.

It is natural to ask whether the single photon piece in eq. 4 is a double counting of $\gamma P P$ vertex, since the vertex can also arise from the minimum coupling of Dirac term. The answer is of course no, as we have learned from the Skyrme lagrangian that such vertex could exit in both places. An explicit way to show that the double counting does not occur is to decompose the single photon piece into two part, a part proportional to $\gamma_{\mu}$ and a part proportional to $\sigma_{\mu \nu}$, and then check whether the $\gamma_{\mu}$ piece contributes to $F_{1}(0)$ in Rosenbluth formular. The normalization of proton charge requires $F_{1}(0)$ remains to be one. We found that indeed the single photon piece does not contribute to $F_{1}(0)$.

Anomalous Magnetic Moment The anomalous magnetic moment of nucleon can not arise from the minimum coupling of Dirac term. Traditionally, the term which gives the the anomalous magnetic moment of nucleon is written in a gauge invariant form 


$$
L=-\frac{\kappa \mu_{B}}{2} \bar{\psi} \sigma_{\mu \nu} F^{\mu \nu} \psi
$$

The anomalous magnetic moment $\kappa$ is left as a free parameter which is to be determined. Many predictions of the anomalous magnetic moment are actually symmetry statement in nature, namely, they are the just simultaneous fittings of the anomalous magnetic moment of baryon octet by using $S U(3)$ relation. So the success of predictions is rather an evidence of hadron symmetry than the understanding of hadron structure. It is the purpose of the present study to predict the anomalous magnetic moment of nucleon by relating it to the nucleon anomaly.

Since we start with a very different approach of constructing lagrangian, it is not transparent to see how can we compare our result with the form in eq. 5. An essential definition of anomalous magnetic moment can be defined through the magnetic structure function of nucleon probed in the differential cross section of electron-nucleon elastic scattering. Using the lagrangian in eq. 4 , it is a simple exercise to calculate the differential cross section of $e^{-} P \rightarrow e^{-} P$

$$
\begin{aligned}
\frac{d \sigma}{d \Omega}= & \left(\frac{d \sigma}{d \Omega}\right)_{M o t t}\left\{\left[1-\frac{q^{2}}{4 M^{2}}\left(\frac{M^{2}}{4 \pi^{2} f_{\pi}^{2}}\right)\right]\right. \\
& \left.+-\frac{q^{2}}{4 M^{2}} \cdot 2\left[1+\left(\frac{M^{2}}{4 \pi^{2} f_{\pi}^{2}}\right)^{2}\right] \tan ^{2} \frac{\theta}{2}\right\}
\end{aligned}
$$

where $\left(\frac{d \sigma}{d \Omega}\right)_{M o t t}$ is what one would get for a spinless point charge. The magnetic structure function of proton can be read off from the second line in the above equation

$$
G_{M}^{P}=\left[1+\left(\frac{M^{2}}{4 \pi^{2} f_{\pi}^{2}}\right)^{2}\right]^{1 / 2}
$$

It is related to the anomalous magnetic moment of proton $G_{M}^{P}{ }^{2}=\left(1+\kappa_{P}\right)^{2}$. Numerically we find $\kappa_{P}=1.77$ which is in excellent agreement with the experimental data $\kappa_{P}=1.79$. The magnetic structure function of neutron can be derived in the same way and it is given by

$$
G_{M}^{N}=-\frac{M^{2}}{4 \pi^{2} f_{\pi}^{2}}
$$


The numerical value $\kappa_{N}=-2.58$ deviates from the experimental data $\kappa_{N}=-1.91$ by $35 \%$, which is less satisfactory as compared with the success of the proton case. Nevertheless, the sign is correct. It is the isovector nature of the axial current coupling leads to the change of sign for the neutron case. No isoscarlar contribution is found from the lagrangian.

We want to stress that the above results are true predictions of hadron structure, since the sole input of this prediction is the relative normalization between pion and nucleon axial currents. This is the main result of the present paper.

Discussion and Conclusion The stratedge of constructing an effective lagrangian is to include all the possible terms allowed by the required symmetries. The conventional form eq. 5 is itself a gauge invariant piece and in principle should be included in the lagrangian at the first sight. But it is redundant to do so as we will see in the following analysis. Using the identity

$$
\epsilon^{\mu \nu \rho \sigma} \gamma_{5} \gamma_{\sigma}=-i \gamma^{\mu} \gamma^{\nu} \gamma^{\rho}+i g^{\mu \nu} \gamma^{\rho}-i g^{\mu \rho} \gamma^{\nu}+i g^{\nu \rho} \gamma^{\mu}
$$

the single photon piece in the lagrangian can be decomposed into two pieces

$$
\begin{aligned}
& \frac{e M}{i 4 \pi^{2} f_{\pi}^{2}} \bar{\psi} \sigma_{\alpha \beta} \frac{\tau^{3}}{2} F_{\alpha \beta} \psi \\
& +\frac{e M}{4 \pi^{2} f_{\pi}^{2}} \bar{\psi} \frac{\tau^{3}}{2}\left[\partial^{\alpha} \psi \partial_{\alpha} \not A-\partial^{\beta} \psi \not \partial A_{\beta}\right] .
\end{aligned}
$$

The first term is gauge invariant by itself and is of the conventional form. So the conventional form has been included implicitly in the lagrangian already. However, it is not proper to identify the coefficient $\frac{e M}{i 4 \pi^{2} f_{\pi}^{2}}$ as $-\kappa \mu_{B}$, because the second term also contributes to the magnetic structure function. Due to the nonrenormalization theorem of anomaly, we conjecture that the coefficients of the lagrangian is not renormalized by quantum correction.

The lagrangian shares various common results with the Skyrme model [4]. The leading contribution to the anomalous magnetic moment is isovector in nature and this leads to the correct sign of the neutron anomalous magnetic moment. However, the W.Z.W. lagrangian in the Skyrme model only gives arise to the isoscalar part of the anomalous magnetic moment 
and it is numerically small compared with the isovector part arising from the kinetic energy term. The source of the difference remains an issue to be further studied.

Replacing the pion current by nucleon current in triangle anomaly, we derived a minimum gauge invariant set of effective lagrangian which describes the anomalous interaction of photons and nucleons. The prediction for the anomalous magnetic moment of proton is in excellent agreement with the experimental data. The construction of the effective lagrangian provides a new way of understanding the hadronic structure of nucleon.

\section{ACKNOWLEDGMENTS}

This research is supported in part by the National Science Council in Taiwan under Contract No. NSC84-2112-M008-013 and NSC84-2732-M008-001. The author likes to thank C. Y. Cheung and S. C. Lee for useful conversation. 


\section{REFERENCES}

1 T. H. R. Skyrme, Proc. R. Soc. London, Ser. A260, 127 (1961).

2 J. Wess and B. Zumino, Phys. Lett.B37, 95 (1971).

3 E. Witten, Nucl. Phys. B223, 422 (1983).

4 E. Braaten, S. Tse and C. Willcox, Phys. Rev. Lett. 56, 2008 (1986). 